\title{
Flow Cytometric DNA Ploidy Analysis of Ovarian Granulosa Cell Tumors
}

\author{
Savi Chadha, M.D., Ph.D., ${ }^{1}$ Cees J. Cornelisse, Ph.D., ${ }^{*}$ and Aart Schaberg, M.D., Ph.D. \\ Department of Pathology, Erasmus University, Rotterdam, The Netherlands, and *Department of Pathology, Leiden University, \\ The Netherlands
}

Received November 29, 1988

The nuclear DNA content of 50 ovarian tumors initially diagnosed as granulosa cell tumors was measured by flow cytometry using paraffin-embedded archival material. The follow-up period of the patients ranged from 4 months to 19 years. Thirty-eight tumors were diploid or near-diploid, while 5 were aneuploid. DNA profiles of 7 tumors could not be evaluated. All 50 tumors were immunohistochemically tested for expression of intermediate filament proteins vimentin and cytokeratin and epithelial membrane antigen. The cells of all but 3 tumors expressed vimentin. These 3 vimentin-negative tumors were positive for cytokeratin and epithelial membrane antigen. They were highly aneuploid and though originally diagnosed as granulosa cell tumors, most likely represent undifferentiated carcinomas. Hence, only 2 typical granulosa cell tumors were aneuploid. In addition, frozen tissue samples from 9 of $\mathbf{1 0}$ granulosa cell tumors showed a DNA diploid content. Our results indicate that granulosa cell tumors tend to be diploid or have only minor ploidy abnormalities which is in line with their relatively benign character. An undifferentiated carcinoma should be considered in the differential diagnosis of tumors with a high DNA index. (c) 1990 Academic Press, Inc.

\section{INTRODUCTION}

Granulosa cell tumors, which account for less than $2 \%$ of all ovarian tumors and less than $10 \%$ of malignant ovarian tumors [1], belong to the category of sexcordstromal tumors.

Together with theca cell tumors they constitute the most common functioning ovarian neoplasms. Most granulosa cell tumors (GCT) can be diagnosed by routine examination of an adequate number of sections stained by hematoxylin eosin. However, it may be difficult to differentiate between poorly differentiated sexcordstromal tumors and undifferentiated carcinomas.

The malignant potential of GCTs is debated $[2,3,4]$. Generally, all GCTs are considered malignant neoplasms

\footnotetext{
' To whom reprint requests should be addressed at Department of Pathology, P.O. Box 1738, 3000 DR Rotterdam, The Netherlands.
}

that may have a prolonged course with a tendency to late recurrence. An association between aneuploidy and aggressive behavior has been demonstrated in epithelial ovarian cancer $[5,6,8,10]$. A similar relationship has been shown in a variety of other cancers [11-15].

In the present paper we report the results of a retrospective study investigating DNA ploidy in GCTs of the ovary.

\section{MATERIALS AND METHODS}

Since 1966, 105 tumors, initially considered to be GCTs, have been registered with the Netherlands Committee for Ovarian Tumors. Follow-up information could be obtained in only 51 of these patients. Ten more cases which had been referred for treatment from the regional hospitals to the Rotterdam Radiotherapy Institute were available for study. The patients ranged in age from 15 to 79 years with a mean age at diagnosis of 49.8 years. Paraffin-embedded tissue blocks were available in 50 cases, which form the basis of this study. In 49 cases material was available from the primary tumor only; in 1 case material from metastatic as well as the primary tumor was available. Follow-up of these patients ranged from 4 months to 19 years with a median follow-up of 3 years and 9 months. All the tumors had been staged according to the recommendation of the International Federation of Gynecology and Obstetrics (FIGO). Additional frozen samples of fresh tumor tissue stored at $-70^{\circ} \mathrm{C}$ were available from 10 cases.

\section{Flow Cytometry (FCM)}

Nuclear DNA content of paraffin-embedded tumor tissue was analyzed by FCM using the method of Hedley et al. [16]. In all cases the paraffin blocks used for DNA measurements were checked for the presence of sufficient tumor tissue by examining the hematoxylin-azofloxin stained sections. In the majority of the cases the 
percentage of tumor cells was approximately $80 \%$, the lower limit being $30 \%$. Thirty-five-micrometer sections were cut from 1 to 3 paraffin blocks of tumor and an adjacent $5-\mu \mathrm{m}$ section for hematoxylin-azofloxin staining. The sections were placed in glass centrifuge tubes and then rehydrated in $100,96,70$, and $50 \%$ ethanol for 10 min each at room temperature after which the tissue was washed in two changes of distilled water and resuspended in $1 \mathrm{ml}$ of $0.5 \%$ pepsin in $0.9 \% \mathrm{NaCl}(\mathrm{pH}$ 1.5). The tubes were placed in a water bath at $37^{\circ} \mathrm{C}$ for 30 min with intermittent vortex mixing. The cell suspension was then centrifuged, supernatant fluid removed, and the pellet suspended in $1 \mu \mathrm{g}$ per $\mathrm{ml} 4^{\prime} 6^{\prime}$-diamidino2-phenylindole dihydrochloride (DAPI) for $45 \mathrm{~min}$ at room temperature and then filtered through nylon gauze. Suspensions of single cell nuclei were prepared from frozen tissue blocks with the detergent-trypsin procedure of Vindelov [17] and stained with propidium iodide. Rainbow trout red blood cells were added to the suspensions of isolated nuclei as an internal ploidy standard.

The stained samples were measured on an ICP 22 flow cytometer (Ortho, Westwood, MA). For excitation of DAPI fluorescence a 365 UGI filter and $400-n m$ chromatic beam splitter were used. Emission was measured using a $435 \mathrm{GG}$ barrier filter. For excitation of propidium iodide fluorescence, LP 515 and SP 560 filters were used in combination with a $560-\mathrm{nm}$ chromatic beam splitter. Emission was measured using a LP 590 barrier filter. Filtered demineralized water was used as a sheath fluid.

\section{Classification of Ploidy Abnormalities}

The ploidy of the tumor was expressed by the DNA index (DI) being the ratio between the modal channel number of the GO, 1 peak of the tumor cell population and that of the GO, 1 peak representing the nonncoplastic cell population present in tumor samples. When the DNA profile showed two different GO,1 peaks the one on the left was considered to represent normal diploid cells.

\section{Histology and Immunohistochemistry}

Histological criteria for diagnosis of GCT. A typical GCT is composed of small round polygonal or spindleshaped cells containing a small amount of cytoplasm with rather indistinct boundaries. The nuclei are usually pale or at most slightly hyperchromatic round ovoid or angular with a well defined limiting membrane and a longitudinal groove resulting in coffee-bean appearance. A single nucleolus is usually present. A variety of histological patterns, microfollicular, insular, trabecular, and diffuse, may be encountered. Call-Exner bodies which are small rounded cavities containing eosinophilic material or pyknotic nuclei may be present. Juvenile GCTs may show hyperchromatic sometimes anaplastic nuclei. Nuclear grooves are rare or absent.

Immunohistochemistry. Immunohistochemistry was performed to demonstrate the presence of the intermediate filament proteins vimentin, cytokeratin, and epithelial membrane antigen.

Paraffin sections (5- $\mu \mathrm{m}$ thick) were deparaffinized in xyelene and then placed in $100 \%$ ethanol. The endogenous peroxidase activity was blocked by immersing the sections in a solution of methanol containing $3 \%$ hydrogen peroxide for $30 \mathrm{~min}$. For detection of keratin and vimentin the sections were then subjected to a poteolytic treatment using $1 \%$ protease (Sigma Chemical Co., St. Louis, MO) in phosphate-buffered saline (PBS) for 25 min at $37^{\circ} \mathrm{C}$. Subsequently, sections were incubated with appropriate dilutions of the primary antibodies for $1 \mathrm{hr}$ at room temperature. Rabbit anti-keratin, rabbit antivimentin were commercially obtained from Eurodiagnostics, DAKO Holland. The sections were finally incubated with peroxidase-conjugated swine anti-rabbit IgG serum or peroxidase-conjugated rabbit anti-mouse IgG antiserum (DAKO, Denmark) 1 in 20 dilution for $30 \mathrm{~min}$. Following each incubation sections were washed thoroughly with PBS. Antibody localization was determined on the basis of peroxidase activity effected after 10-min incubation of the sections with a TRIS-buffered saline solution $(0.05 M, \mathrm{pH} 7.4)$ containing $50 \mathrm{mg} \% 3,3$ diaminobenzidine-tetrahydrochloride (Fluka, Federeal Republic of Germany) and $0.03 \%$ hydrogen peroxide.

Slides were then washed with water, counterstained with hematoxylin, and mounted in Malinol.

\section{RESULTS}

\section{Flow Cytometry}

DNA profiles with only a single GO,1 $(\mathrm{DI}=1.0)$ peak were obtained for 38 archival deparaffinized tumor specimens, whereas in 5 cases 2 distinct GO,1 populations were found. The latter were classified as aneuploid, considering the peak with the highest modal fluorescence value as the tumor stemline. The DNA indices of the 5 aneuploid tumors were $1.16,1.20,1.49,1.81$, and 1.84 , respectively. The 38 cases with a single GO,1 peak showed a considerable variation of the coefficient of variation (CV) which ranged from 2.4 to $13.7 \%$ (mean 9.6\%). A positive correlation was found between the $\mathrm{CV}$ and the age of the blocks (rank correlation coefficient of Spearman $P=0.01$ ). In contrast, low CVs were found for the GO, 1 peaks from 9 out of 10 frozen specimens (1.7 to $3.0 \%$ ). With the range of CVs found for the archival specimens it is not possible to discriminate between tumors with a true diploid DNA content and those with minor ploidy abnormalities. Therefore all DNA pro- 

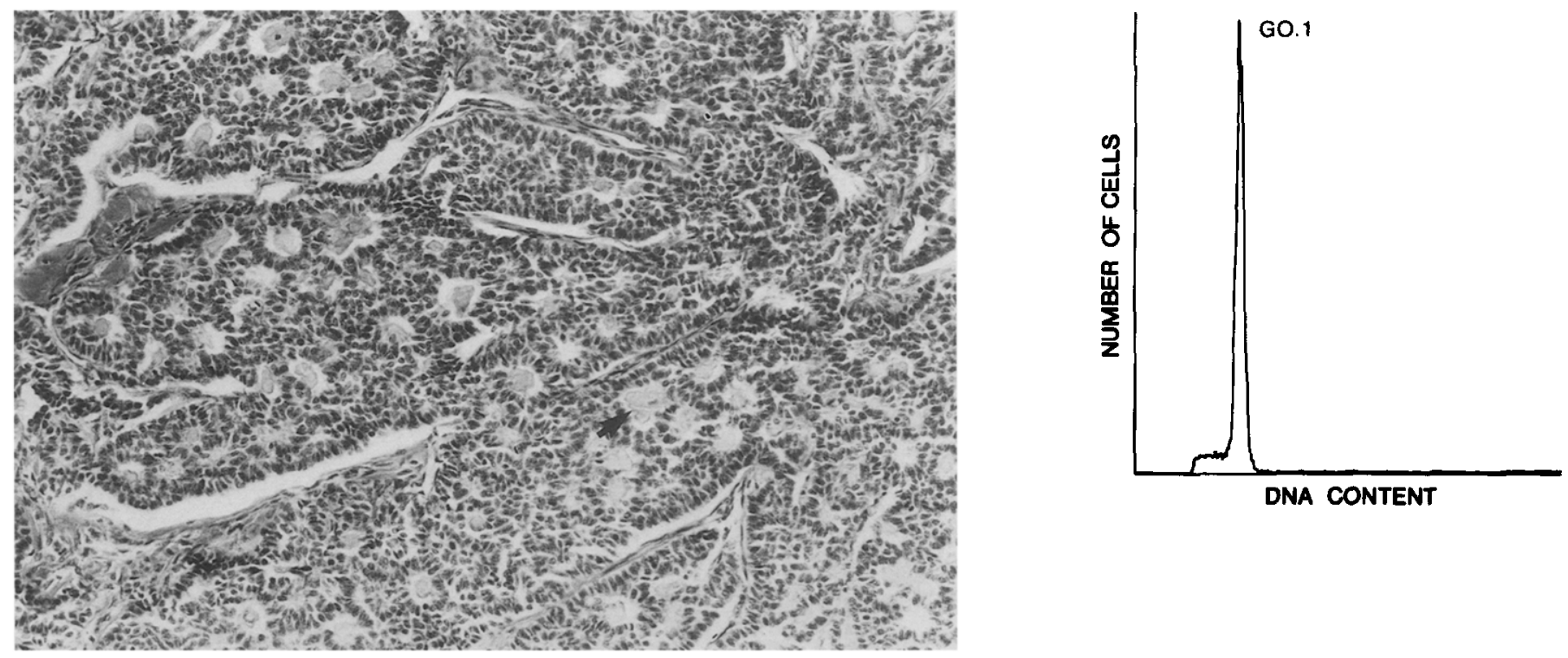

FIG. 1. Near-diploid DNA profile with low CV (3.4\%) of a paraffin-embedded granulosa cell tumor showing microfollicular pattern with Cell-Exner bodies (arrow).

files fom deparaffinized archival specimens showing only a single $\mathrm{GO}, 1$ peak have been classified as (near-)diploid. Frozen samples from 9 out of 10 granulosa cell tumors could be clearly classified as DNA diploid, with only 1 tumor low aneuploid. In Fig. 1 the (near-)diploid DNA profile $(\mathrm{CV}=3.4 \%)$ of a paraffin-embedded granulosa cell tumor with a microfollicular pattern and Call-Exner bodies is shown. Figure 2 shows a (near-)diploid DNA profile of a granulosa cell tumor $(\mathrm{CV}=9.0 \%)$ with a diffuse histological pattern and coffee-bean nuclei. DNA profiles of a primary granulosa cell tumor paraffin embedded showing a diffuse arrangement of granulosa cells (a) and its metastasis (b) both with (near-)diploid stemlines are presented in Fig. 3. Figure 4 shows the aneuploid DNA profile and histology of 1 of the 3 vimentin-negative keratin and EMA positive paraffin-embedded tumors lacking typical pale coffee-bean nuclei.

In 7 cases the DNA profile showed a debris continuum
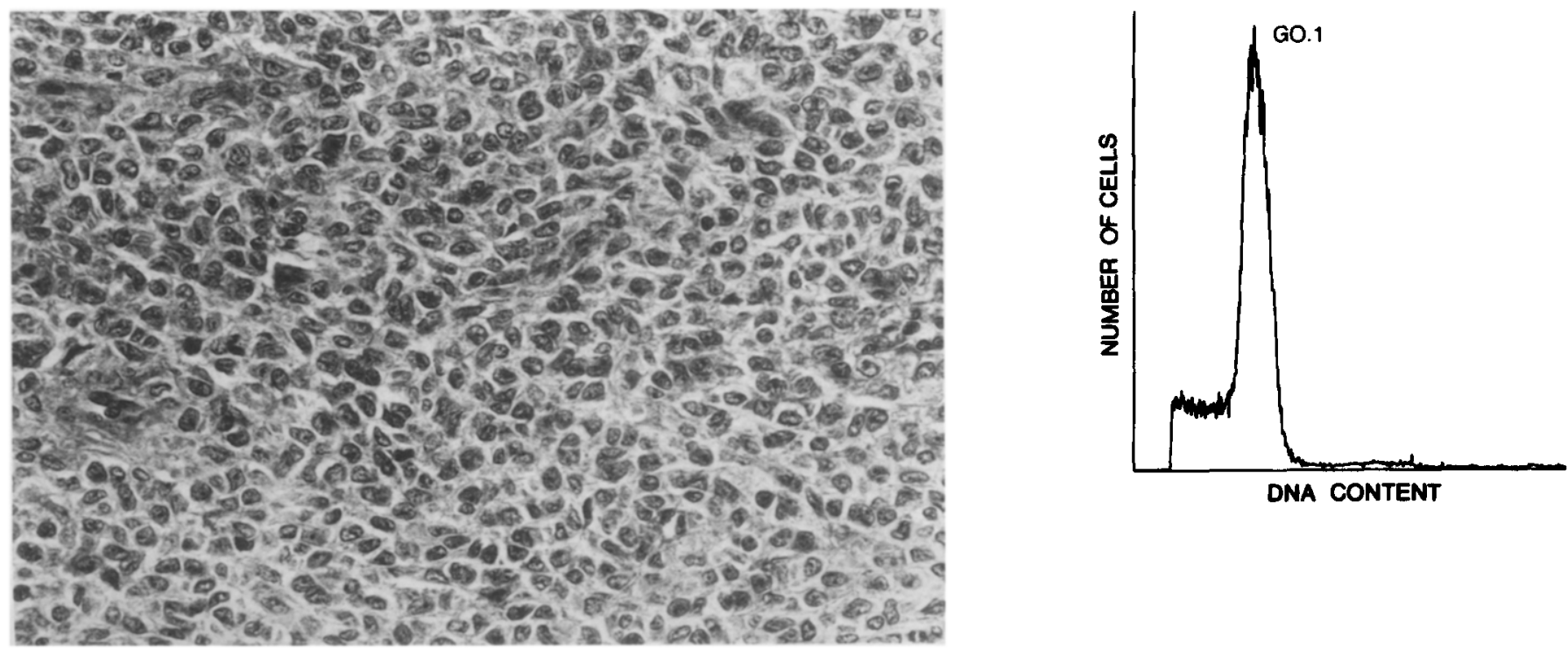

FIG. 2. Near-diploid DNA profile with high CV $(9.0 \%)$ of a paraffin-embedded granulosa cell tumor and a diffuse pattern showing coffeebean nuclei. 

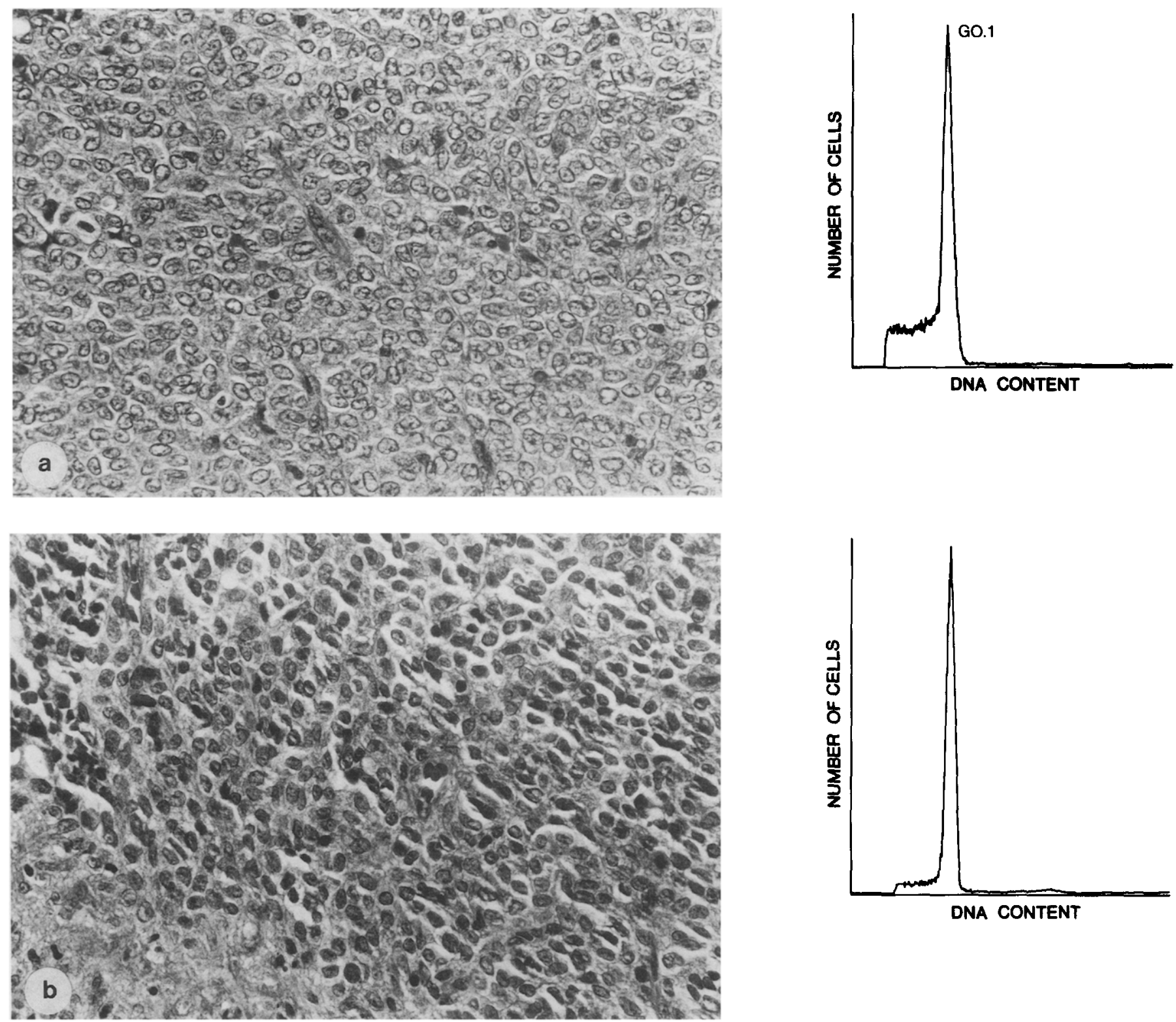

FIG. 3. (a) Near-diploid DNA profile of diffuse type of paraffin-embedded granulosa cell tumor (CV $=4.3 \%$ ). (b) Near-diploid DNA profile of metastasis from tumor in Fig. 3a $(\mathrm{CV}=5.3 \%)$.

only and could not be evaluated. Table 1 summarizes the tumor DNA ploidy results of paraffin-embedded tumor tissue from 43 patients.

\section{Histology and Immunohistochemistry}

Histological sections of 47 of the 50 tumors showed the typical appearances of GCT while 3 neoplasms were atypical. These 3 tumors showed solid areas with included spaces resembling Call-Exner bodies but the typical pale coffee-bean nuclei were replaced by dark hyperchromatic nuclei.
Cells of 47 tumors with typical histological appearance of GCT expressed vimentin while the 3 neoplasms with atypical histological appearance were vimentin negative, cytokeratin and EMA positive. This combination of findings is not consistent with a diagnosis of granulosa cell tumor. Therefore these 3 tumors should be considered undifferentiated carcinomas.

Staging. Table 1 shows the FIGO stage distribution of 43 cases in relation to DNA ploidy. DNA profiles of 7 cases could not be evaluated. The relatively larger proportion of cases of GCT in higher stages as compared to the figures reported in literature could possibly be 

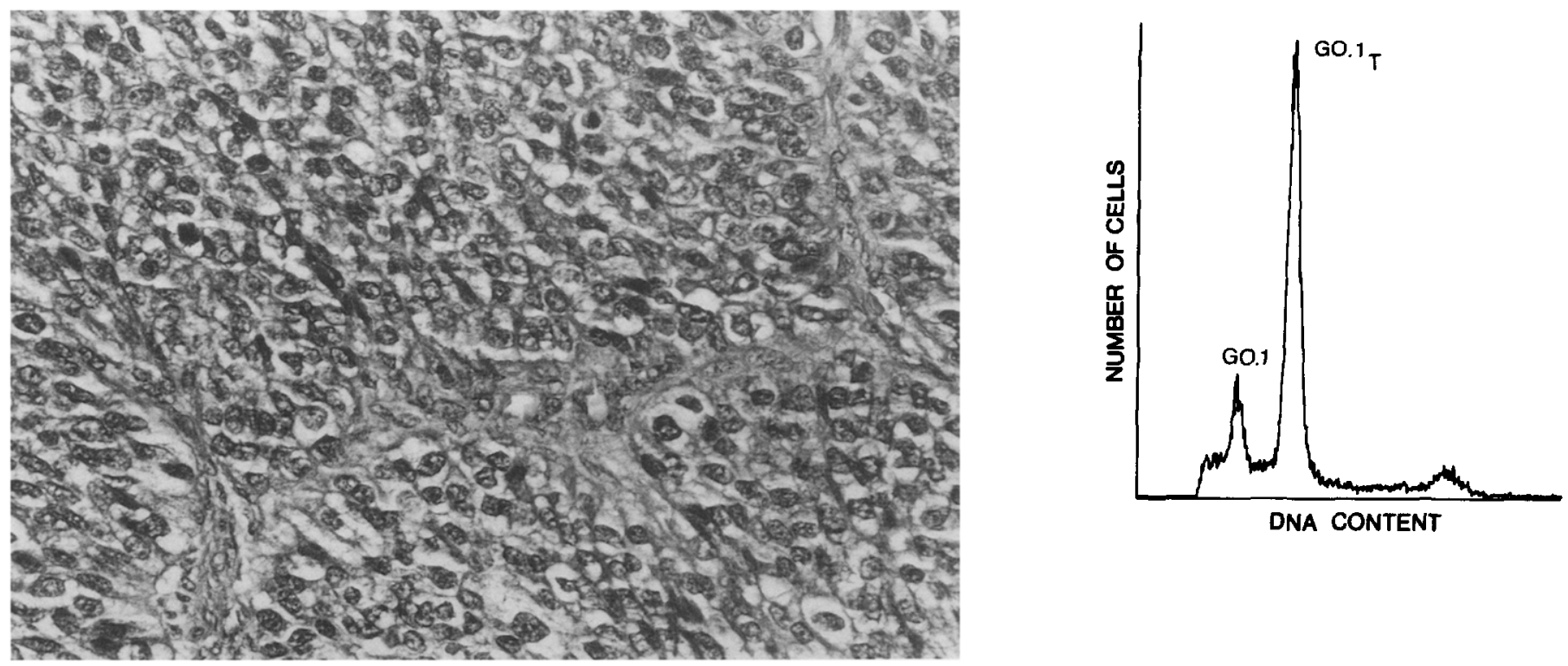

FIG. 4. Aneuploid DNA profile of a parafiin-embedded undifferentiated carcinoma lacking coffee-bean nuclei. GO,1 = aneuploid GO,1 fraction.

explained by a bias in the material referred to the Netherlands Committee for Ovarian Tumors.

Survival. Out of 38 patients with (near-)diploid tumors with the typical features of GCT, 31 are still alive. In contrast 1 patient out of 5 with aneuploid tumors (2 typical GCT, 3 carcinomas) is alive. A high CV was not related to survival in the (near-)diploid group. The 7 patients with typical and (near-) DNA diploid GCT who died were in different FIGO stages: 3 had stage I, 2 stage II, and 2 stage IV disease. Two of the patients with stage I GCT died due to other causes (lung cancer and heart disease). The other 5 patients dued due to recurrence of GCT.

Of 5 patients with aneuploid tumors 1 had stage I disease, 1 stage IIb, 1 stage III, and 2 stage IV disease. Four patients from this group died of their malignant disease. The remaining one patient with stage I disease and typical GCT was lost to follow-up after 4 years.

TABLE 1

Ploidy Distribution in Relation to FIGO Stage

\begin{tabular}{lccc}
\hline Stage & $\begin{array}{c}\text { Diploid-(peri)diploid } \\
(38 \text { cases }) \\
\text { GCT }\end{array}$ & $\begin{array}{c}\text { Aneuploid } \\
(2 \text { cases }) \\
\text { GCT }\end{array}$ & $\begin{array}{c}\text { Aneuploid } \\
(3 \text { cases }) \\
\text { carcinoma }\end{array}$ \\
\hline I & $24(63 \%)$ & $1(50 \%)$ & \\
II & $6(16 \%)$ & & $1(33.3 \%)$ \\
III & $4(10.5 \%)$ & $1(50 \%)$ & $1(33.3 \%)$ \\
IV & $4(10.5 \%)$ & DNA index & DNA index \\
& DNA index & $1.16-1.20$ & $1.16-1.84$ \\
\hline
\end{tabular}

\section{DISCUSSION}

In our series, out of 43 tumors primarily diagnosed as GCTs, 3 have been reclassified as undifferentiated carcinomas leaving 40 GCT. Thirty-eight of these 40 (95\%) GCTs were (near-)diploid. This is in striking contrast to the results reported for ovarian carcinomas $[5,9,10]$ : Friedlander et al. [5] found $76 \%$ aneuploidy versus $24 \%$ diploidy and Rodenburg et al. [10] $61 \%$ aneuploidy versus $39 \%$ (near-)diploidy. Volm et al. [9] in their series reported $71 \%$ aneuploidy versus $29 \%$ diploidy. In cancers of various organs diploidy is associated with a significantly better prognosis as compared to DNA aneuploid tumors. Friedlander et al. [5], Volm et al. [9], and Rodenburg et al. [10] have shown that DNA ploidy is an important prognostic indicator in epithelial cancer. It is known that granulosa cell tumors of the ovary have a low malignant potential with a favorable prognosis and a tendency to late recurrence.

Thus the predominance of (near-)diploid stemlines seems to be compatible with and perhaps related to the known relatively favorable prognosis of these tumors. In our series the follow-up period was rather short for determining the prognosis of GCT with a median of 3 years and 9 months. Nevertheless the survival data of this group of GCT are compatible with the general relatively benign course of diploid tumors considering that 31 out of 38 patients with a (near-)diploid tumor are still alive.

Out of the 5 aneuploid tumors 3 reclassified as undifferentiated carcinomas showed the highest DNA ploidy (DI $1.49,1.81,1.84)$. These 3 cases also had a poor survival since the 3 tumors became fatal in 3 years. In 
addition, at diagnosis these 3 patients were in a higher stage (IIb, III, IV) which is comparable with previous observations on epithelial ovarian cancer showing that diploid tumors tend to be low stage while aneuploid ones are of high stage [5].

The remaining 2 aneuploid tumors had lower DNA indices $(1.16,1.20)$. They showed typical histological appearances of GCT; they were vimentin positive and keratin as well as EMA-negative. One of these patients died within 8 months with metastases (stage IV); the other patient was lost to follow up after 4 years (stage I). Unfortunately the numbers in our series are too small to be able to derive any conclusions regarding the ploidy prognosis relationship.

In these series tumors classified as (near-)diploid had a large range of CVs, those with a high $\mathrm{CV}$ indicating perhaps the possibility of low aneuploidy ( $2 \mathrm{GO}, 1$ peaks merged in 1 leading to a high CV). However, no relation was found between a high $\mathrm{CV}$ and survival. Another possible explanation of the high CVs may possibly be found in the age of the paraffin blocks, some of the blocks being as old as 19 years. Considering that the paraffinembedded material was obtained from different laboratories, fixation of tumor tissue may have been substandard in a number of cases, e.g., by using unbuffered formalin which could lead to DNA loss by acid hydrolysis.

In conclusion the results of our study of ovarian GCTs indicate that (a) the majority of these neoplasms is diploid or has minor ploidy abnormalities not detectable by flow cytometry on deparaffinized tissue sections. This was substantiated by our results on frozen samples of GCTs which were clearly DNA diploid in 9 cases and low DNAaneuploid in 1 case, (b) An undifferentiated carcinoma should be considered in the differential diagnosis of tumors with a high DNA index, (c) The predominant DNA diploid nature of ovarian granulosa cell tumors is in line with their low malignant biological behavior.

\section{ACKNOWLEDGMENTS}

We thank Professor Dr. R. O. van der Heul for his scientific support and Dr. G. J. Fleuren for his contribution to this study. We are also grateful to Mrs. N. J. Dijkshoorn for performing FCM and Mrs. M. Hanegraaff for preparing the manuscript.

\section{REFERENCES}

1. Scully, R. E. Tumors of the ovary and maldeveloped gonads, in Atlas of tumor pathology, Armed Forces Institute of Pathology, Washington, DC, 2nd series, Fascicle No. 16 (1980).
2. Fox, H., Agrawal, K., and Langley, F. A. A clinicopathological study of 92 cases of granulosa cell tumor of the ovary with special reference to factors influencing prognosis, Cancer 35, 213-241 (1975).

3. Norris, H. J., and Taylor, H. B. Prognosis of granulosa theca tumors of the ovary, Cancer 21, 255-263 (1968).

4. Bjorkholm, E., and Silfversward, C. Prognostic factors in granulosa cell tumors, Gynecol. Oncol. 11, 261-274 (1981).

5. Friedlander, M. L., Taylor, I. W., Russel, P., Musgrove, E. A., Hedley, D. H., and Tattersal, M. H. N. Ploidy as a prognostic factor in ovarian cancer, Int. J. Gynecol. Pathol. 2, 55-63 (1983).

6. Friedlander, M. L., Hedley, D. W., Taylor, I. W., Russel, P., Coates, A. S., and Tattersal, M. H. N. Influence of cellular DNA content on survival in advanced ovarian cancer, Cancer Res. 44, 397-400 (1984).

7. Friedlander, M. L., Taylor, I. W., Russel, P., and Tattersal, M. H. N. Cellular DNA content-A stable feature in epithelial ovarian cancer, Brit. J. Cancer 49, 173-179 (1984).

8. Erhardt, K., Auer, G., Bjorkholm, E., Forsslund, G., Moberger, B., Silfversward, G., Wicksell, G., and Zetterberg, A. Prognostic significance of nuclear DNA content in serous ovarian tumors, Cancer Res. 44, 2198-2202 (1984).

9. Volm, M., Bruggeman, A., Gunther, M., Kleine, W., Pfeiderer, A., and Vogtschaden, M. Prognostic relevance of ploidy, proliferation and resistance-predictive tests in ovarian carcinoma, Cancer Res. 45, 5180-5185 (1985).

10. Rodenburg, C. J., Cornelisse, C. J., Heintz, P. A. M., Hermans, J., and Fleuren, G. J. Tumor ploidy as a major prognostic factor for survival in advanced ovarian cancer, Cancer 59, 317-323 (1987).

11. Gustafson, H., Tribukait, B., and Esposti, P. L. DNA profile and tumor progression in patients with superficial bladder tumors, Urol. Res. 10, 13-18 (1982).

12. Wolley, R. C., Schreiber, K., Koss, L. G., Karas, M., and Sherman, A. DNA distribution in human colonic carcinomas and its relationship to clinical behaviour, J. Natl. Cancer Inst. 69, 15-22 (1982).

13. Auer, G., Ericksson, E., Azavedo, E., Caspersson, T., and Wallgren, A. Prognostic significance of nuclear DNA content in mammary adenocarcinomas in humans, Cancer Res. 44, 394-396 (1984).

14. Hedley, D. W., Rugg, C. A., Ng, A. B. P., and Taylor, I. W. Influence of cellular DNA content of disease-free survival of stage II breast cancer patients, Cancer Res. 44, 5395-5398 (1984).

15. Cornelisse, C. J., Van de Velde, C. H. J., Caspers, R. J. C., Molenaar, A. J., and Hermans, J. DNA ploidy and survival in breast cancer patients, Cytometry 8, 225-234 (1987).

16. Hedley, D. W., Friedlander, M. L., Taylor, I. W., Rugg, C. A., and Musgrove, E. A. Method for analysis of cellular DNA content of paraffin embedded pathological material using flow cytometry, J. Histochem. Cytochem. 31(11), 1333-1335 (1983).

17. Vindelov, L. L., Christensen, I. J., and Nissen, N. I. A detergent trypsin method for the preparation of nuclei for flow cytometric analysis, Cytometry 3, 323-327 (1983). 